\title{
The effect of size on the perception of ambiguous figures
}

\author{
PAULA GOOLKASIAN \\ University of North Carolina, Charlotte, North Carolina
}

\begin{abstract}
The effect of size on the interpretation of ambiguous figures was studied. Small (less than $10^{\circ}$ of visual angle) and large (greater than $15^{\circ}$ of visual angle) figures were preceded by a fixation point, a biased version of a figure, or a feature of a figure. Although the interpretation of a large ambiguous figure was found to be influenced by a critical feature or a biased version of a figure, the interpretation of a small ambiguous figure was not affected by the information that preceded it. This finding adds retinal size as another factor to be considered in the explanation of the processing of ambiguous figures.
\end{abstract}

Ambiguous figures are multistable visual stimuli that are perceived in at least two different ways. Although the factor or factors that determine the multistability are unknown at present, both attention to critical features and context effects have been shown to be involved.

Tsal and Kolbet (1985), using the bird/plane and duck/ rabbit figures, measured reaction times to letters placed in the focal area of one of the two alternate figures. Responses were faster when the letters appeared in the focal area of the perceived interpretation rather than in the alternate focal area. Eye-movement studies have also shown that attention to critical features is an important factor. Ellis and Stark (1978) reported that eye fixations are correlated with shifts in the interpretation of ambiguous figures.

The evidence is not unequivocal, however. In one study, when attention was directed to the focal area of a specific interpretation of an ambiguous figure through the placement of a fixation point, the subject's organization of the figure was unaffected by the manipulation (Goolkasian, 1987). Moreover, a second study showed that an ambiguous figure was affected by the advanced presentation of a biased drawing and only slightly influenced by the advanced presentation of a critical feature. These data were consistent with findings in other studies, which showed the relative importance of context effects and top-down processing (Bugelski \& Alampay, 1961). Interpretations of the figures were influenced more by a preview of the overall context of the figure than by the presence of a specific feature that directed attention to one of the two focal areas.

Recently, García-Pérez $(1988,1989)$ has suggested that the absence of an effect of the fixation point might result from a failure to consider the retinal size of the ambiguous figure and the role of visual inhomogeneity. It is well

Thanks are due to Jailaxmi Desai for her assistance with this project. Requests for reprints should be sent to Paula Goolkasian, Department of Psychology, University of North Carolina, Charlotte, NC 28223. known that the quality of visual processing varies with changes in resolution. Since fine detail is available only within a small area around the fixation point, the fixation location of a large stimulus would determine which detail information was available, and eye movements would correlate more closely with alternation in perception. With a small stimulus, however, the critical features for both interpretations of a figure may be visible at a glance, and eye movements may not necessarily correlate with alternation in perceptions of the figure. This hypothesis is consistent with studies in which stimulus size has been found to be important for obtaining a global advantage in compound stimuli made up of local and global features. The dominance of global over local processing has been found to depend on the size of the compound stimuli (Kinchla \& Wolfe, 1979). A global advantage was reported for stimuli of less than $10^{\circ}$ of visual angle, but when letters were larger, responses to local elements were faster than to global elements. However, Navron and Norman (1983) reported that this finding resulted partially from a confounding with eccentricity. When eccentricity was kept constant, global features were responded to faster than local features, over a wide range of visual angles.

My purpose in the present study was to look at whether the size of an ambiguous figure would influence a subject's interpretation. Small and large ambiguous figures appeared briefly $(200 \mathrm{msec})$, but they were preceded by one of four types of stimuli: (1) a fixation point positioned at the spatial location of a critical feature of one of the two possible interpretations of the figure, (2) a biased version of the ambiguous figure, (3) a critical feature of one of the two possible figures, and (4) a neutral feature of the figure, or a different feature taken from another figure. If size has an influence on the manner in which ambiguous figures are processed, then I expected that the size of the stimulus would interact with the type of information presented first. Support for the García-Pérez notion of visual inhomogeneity would be found if the interpretation of large figures were found to be affected more by the advanced presentation of a critical feature or of a fix- 
ation point that directs attention to the focal area of one of the versions of the ambiguous figure than by the biased versions of the figure. Presentation of a neutral or a different feature of the ambiguous figure was used as a control condition, to determine the subject's interpretation of the figure in the absence of a biasing element. Performance in this condition was expected to be at chance. Comparisons were made between this condition and the others, to assess the relative influence of each of the experimental conditions.

\section{METHOD}

\section{Materials and Apparatus}

The four ambiguous figures used in this study were the same as in Goolkasian (1987). The bird/plane and the duck/rabbit figures were reproduced from the drawings of Tsal and Kolbet (1985). The man/ woman examples were taken from a set of drawings provided by Fisher (1967) and the rat/man figures were reproduced from Chastain and Burnham (1975).

On each trial, one of four types of stimulus information preceded the presentation of the ambiguous figure. A biased version of each figure was taken from the same source as the ambiguous figure was. For all of the figures, the ambiguous drawings represented a figure in which at least two alternate interpretations were reported to be equiprobable, and the biased versions represented examples in which only one interpretation was seen as dominant. Figure 1 presents the ambiguous and biased versions of the figures used in this study.

Critical features represented a dominant feature of one of the two alternate organizations of the ambiguous figure. These features, which had been used in previous studies, were reproduced from the biased figures by masking the surrounding stimulus elements. Neutral features

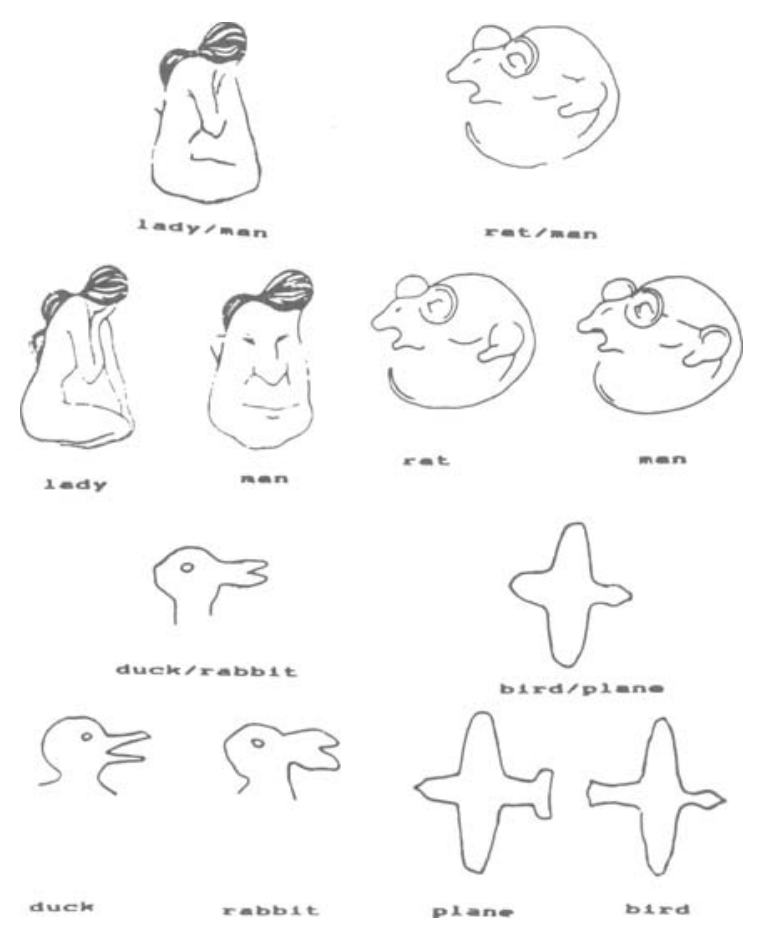

Figure 1. Ambiguous and biased version of each figure. (Journal of General Psychology, 114(3), 217-228, 1987. Reprinted with permission of the Helen Dwight Reid Educational Foundation. Published by Heldref Publications, 4000 Albemarle St. N.W., Washington, DC 20016. Copyright (C) 1987.)
Table 1

Stimulus Features for Each of the Experimental Conditions

\begin{tabular}{lllll}
\hline & & \multicolumn{3}{c}{ Feature Condition } \\
\cline { 3 - 3 } \multicolumn{1}{c}{ Figure } & Response & Critical & Neutral & Different \\
\hline lady/man & lady & thigh & hair & cockpit \\
lady/man & man & nose & & \\
rat/man & rat & tail & nose & beak \\
rat/man & man & eyeglasses & & \\
bird/plane & bird & head & wings & eyeglasses \\
bird/plane & plane & cockpit & & \\
duck/rabbit & duck & beak & eye & tail \\
duck/rabbit & rabbit & ears & & \\
\hline
\end{tabular}

represented features present in both interpretations of the figure, and different features were instances in which features not present in the ambiguous figure appeared. The stimuli in this condition were randomly selected from features of other figures. Also, when features of the stimuli appeared, a word was added to the bottom of the slide to describe the feature. Table 1 presents the words that were used. The figures and the features were enlarged with a copy machine and made into slides, using LPD 40 film. The fixation point was a black dot positioned so that it appeared at the same spatial location as a critical feature of the ambiguous figure.

The stimuli were slides rear-projected on a screen by means of three Kodak Carousel slide projectors with tachistoscopic shutters (Gerbrands). One projector was used to present the ambiguous figures, while the second was used for the presentation of the advanced information. The third presented a visual mask consisting of rows of Xs that covered the visual field. Vocal response times were recorded by a voice key (Gerbrands) and an accompanying digital timer/counter. BRS/LVE electromechanical control panels were used to synchronize the presentation of the slides on each trial and to start the timer.

\section{Subjects and Procedure}

The subjects were 28 men and women student volunteers from the University of North Carolina at Charlotte who had normal or corrected to normal 20/20 vision. They participated in the experiment to obtain extra credit points toward their psychology class grade.

The students participated in the experiment individually in 20-min sessions. They were seated $46 \mathrm{~cm}$ from the screen, and they used a chinrest to stabilize their head movements during the experiment. Before participating in the experiment, the subjects were familiarized with both ambiguous and biased versions of each figure and they participated in two practice trials with the voice key.

On each trial, after a ready signal from the experimenter, a fixation point, a feature of a figure, or a biased figure appeared for $1.5 \mathrm{sec}$; this was followed immediately by an ambiguous figure for $200 \mathrm{msec}$. Upon offset of the figure, a visual mask appeared and remained on the screen until the next trial. The subjects were instructed to interpret the figure presented on the second slide as quickly as they could by speaking into the voice key. The timer was started by the presentation of the second slide and stopped by the subject's vocal response.

The subjects participated in 32 trials, half with each of the two sizes of stimuli. The stimuli were varied in size (small and large) through the use of two projection distances-27.9 and $203 \mathrm{~cm}$. At the closer projection distance, the widh and height of the ambiguous figures varied from $3.7^{\circ}$ to $6.8^{\circ}$ of visual angle. At the farther distance, the ambiguous figures projected a figure that varied in width and height within the range of $17.5^{\circ}$ to $29.5^{\circ}$ of visual angle. Stimuli from the four experimental conditions were similarly scaled in size.

Two sets of 16 pairs of slides were used. A set consisted of each ambiguous figure presented four times paired with a slide representing one of the four experimental conditions-fixation point, critical feature, biased figure, or neutral (different) feature. The experimental conditions represented in the two sets of slides were matched, so that, for example, if the lady/man ambiguous figure was presented with the biased version of the lady in Set 1 , Set 2 would have the lady/man with the biased version of the man. The presentation order of the ambiguous figures and of the experimental conditions within each set was random, 
with the restriction that the same ambiguous figure would not be presented twice in succession. The starting place for each set of slides was varied for each subject.

Each subject was shown a set of stimuli at each of the two projection distances, and the stimulus sets were presented in counterbalanced order across subjects.

\section{RESULTS}

The data are proportions for each subject across the four figures tested within each of the experimental conditions. In the neutral condition, the proportion represents the rate at which the subject reported one of the two possible interpretations of the figures. This measure was transformed into radians according to the following formula: $Y^{\prime}=$ $2 \arcsin \sqrt{Y}$, where $Y$ represents the raw data. For proportions equal to zero or one, the formula was modified as follows: $Y^{\prime}=2 \arcsin \sqrt{Y_{ \pm .03}}$. An angular transformation such as this is recommended with proportion data, to correct for nonnormality and heterogeneity of variance (Kirk, 1982). The transformed data were treated with an analysis of variance to test for the within-subject effects of stimulus size and experimental condition, as well as for their interaction. A comparison of the analyses conducted on the proportions before and after the angular transformation showed that the results of the analyses were comparable, and when the original data are plotted, they show the same trends as those in the transformed data. Only the analysis on the transformed data will be discussed further.

The kind of information presented on the first slide had a significant impact on the subjects' perception of the ambiguous figures $[F(3,81)=4.54, p<.005]$, and there was a marginally significant interaction between this variable and the size of the stimulus $[F(3,81)=2.38, p<$ .07]. However, there was no main effect of stimulus size $(F<1)$. Figure 2 presents the interaction of stimulus size with experimental condition. (The transformed data are

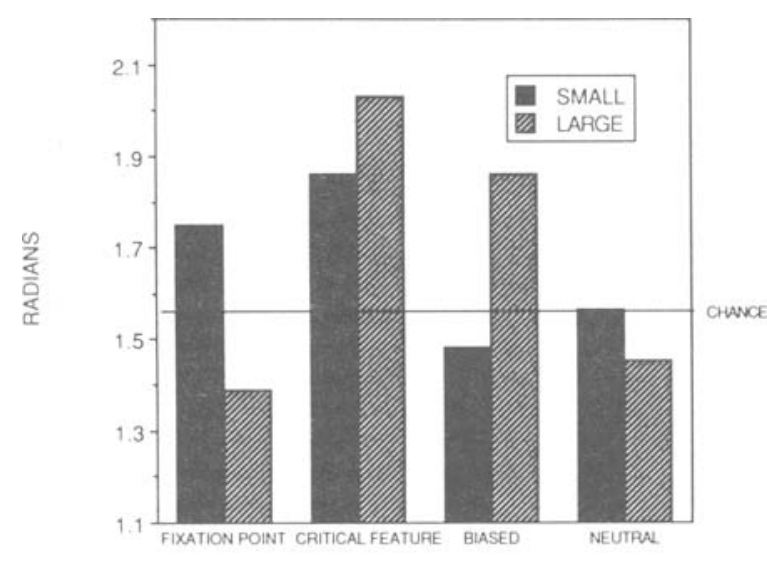

EXPERIMENTAL CONDITION

Figure 2. Mean proportions of times figures identified in accord with information presented on the first slide. (The data presented are transformed scores.) radians, with higher scores indicating better performance. Performance at chance would correspond to a radian value of 1.57.)

To understand the interaction, a test for the simple effect of experimental condition at each of the two stimulus sizes was done. The results show that, although the kind of information presented on the first slide had a significant impact on the interpretation of the large ambiguous figures $(p<.0001)$, its impact on the small ambiguous figures was insignificant $(p=.24)$. For the large figures, a Scheffé test showed that the experimental conditions fell into two groups, with means for the biased and critical feature conditions significantly higher than means for the other two conditions ( $p s<.05)$. When the stimuli were large, the subjects' interpretations of the ambiguous figures were biased by the presentation of critical features of the ambiguous figures or by biased versions of the figures, relative to trials on which neutral features were presented. As expected when neutral features or features from a different figure appeared first, performance was at chance. A comparison of the results from the neutral features and the different features showed comparable performance for each of the four figures.

However, presentation of a fixation point that directed attention to the focal area of one of the two alternate organizations of the stimulus patterns did not affect the subjects' perception of the large ambiguous figures. Performance in this condition did not differ from performance in the neutral condition.

When small ambiguous figures appeared, the kind of information that was presented first did not have a significant impact on the perception of the ambiguous figures. Although Figure 2 does show that presentation of a critical feature of a stimulus or a fixation point that directs attention to a specific feature of a stimulus does have some positive effect on perception, the effect was not found to be significantly different relative to the neutral condition.

\section{DISCUSSION}

\footnotetext{
The results of this study suggest that size has some influence on how subjects organize an ambiguous figure. Figures that exceed $15^{\circ}$ of visual angle were readily influenced by the stimulus information that preceded them. And it did not matter whether that information was simply a critical feature of the ensuing figure or a biased version of the whole figure. When features that are neutral with regard to the alternate organizations of the figure, or that are unrelated to the figure, are presented first, interpretations are at chance; that is, each of the two interpretations is equally likely. The interpretations of small ambiguous figures were not found to be influenced by the information that preceded it.

Although this finding is consistent with García-Perez's prediction regarding the role of retinal size in the interpretation of ambiguous figures, the data do not suggest that visual inhomogeneity is the factor that is producing the size effect. Were that the case, then directing attention to the focal area of one of the two possible interpretations should have been sufficient to influence the interpretation of a large figure. In this study, presentation of a fixation point in a specific area of a large figure was not found to influence the subject's perception of that stimulus pattern. Presentation of the fixation point in a specific part of that figure had a small but insignificant effect on interpretation of small ambiguous figures. In comparison, advanced presentation of the critical feature itself had a substantial impact on organization of the ambiguous figure. Also, arguing against the mechanism of visual inhomogeneity
} 
as an explanation of the tendency to interpret large ambiguous patterns in certain ways is the fact that presentation of the biased version of the stimulus pattern had a substantial impact on organization of the ambiguous figures but only when the figures were large. These findings add retinal size as another important factor to be considered in the explanation of how ambiguous figures are processed.

\section{REFERENCES}

Bugelski, B. R., \& Alampay, D. A. (1961). The role of frequency in developing perceptual sets. Canadian Journal of Psychology, 15, 205-211.

Chastain, B., \& Burnham, C. A. (1975). The first glimpse determines the perception of an ambiguous figure. Perception \& Psychophysics, 17, 221-224.

Ellis, S. R., \& Stark, L. (1978). Eye movements during the viewing of the Necker Cube. Perception, 7, 575-581.

Fisher, G. H. (1967). Preparation of ambiguous stimulus materials. Perception \& Psychophysics, 2, 421-422.
GarcíA-PÉrez, M. A. (1988). Space-variant visual processing: Spatially limited visual channels. Spatial Vision, 3, 129-142.

GarCía-Pérez, M. A.(1989). Visual inhomogeneity and eye movements in multistable perception. Perception \& Psychophysics, 46, 397-400.

Goolkasian, P. (1987). Ambiguous figures: Role of context and critical features. Journal of General Psychology, 114, 217-228.

KinCHLA, R. A., \& WolfE, J. M. (1979). The order of visual processing: "Top-down," "bottom-up," or "middle-out." Perception \& Psychophysics, 25, 225-231.

KIRK, R. E. (1982). Experimental design (2nd ed.). Belmont, CA: Brooks/Cole.

Navon, D., \& Norman, J. (1983). Does global precedence really depend on visual angle? Journal of Experimental Psychology: Human Perception \& Performance, 9, 955-965.

TSAL, Y., \& KolBET, L. (1985). Disambiguating ambiguous figures by selective attention. Quarterly Journal of Experimental Psychology, 12, 97-136.

(Manuscript received October 15, 1990.) 